\title{
Simulation and Optimization of a Permanent Magnet for Small-sized MRI by Genetic Algorithm
}

\author{
Yiyuan Cheng $^{1, a}$, Ling Xia ${ }^{1, b}$ and Wei He ${ }^{1, c}$ \\ ${ }^{1}$ Department of Biomedical Engineering, Zhejiang University,Hangzhou, China \\ achengyy850@gmail.com, b xialing@zju.edu.cn, c violahw@126.com
}

Keywords: simulation; optimization; permanent magnet; genetic algorithm

\begin{abstract}
The main magnet produces the main magnetic field in the imaging area as one of the important parts of the magnetic resonance imaging (MRI) system. In a permanent MRI magnet, the widespread end effect causes a non-uniform magnetic field distribution and affects the imaging quality. In this paper, an H-type permanent magnet for small-sized MRI applications was designed; in particular, we added an optimized shimming ring outside the pole piece to improve the magnetic field uniformity. Genetic algorithms are used to solve the complex and nonlinear calculation of the magnetic field. The simulation results show that the magnet optimized by the proposed method generates a homogeneous magnetic field that can be easily implemented in practice.
\end{abstract}

\section{Introduction}

MRI is an important medical diagnostic imaging modality. The configuration of the magnet assembly is one of the most crucial, and the most expensive aspects determining the quality of the MRI device [1].

There are three broad types of magnets: resistive, permanent and superconducting. Resistive magnets have been gradually phased out. Superconducting magnets have become more popular because of the high intensity and uniformity of the field. However, they are very expensive to purchase, install and run and require liquid helium for cooling. Although permanent magnets generate a lower intensity and poorer homogeneity compared to superconducting magnets, their essential advantages are a lower consumption of electricity, requiring no coolant water or expensive liquid helium and lower cost. In recent years, permanent magnet assemblies have been applied to the manufacturing of the MRI for wrist, ankle and so on, in addition to whole human body systems. These magnets are being widely used in the low-end medical and animal experimental MRI and have a large market demand [2].

The widespread end-effect of permanent magnets always causes a non-uniform magnetic field distribution and affects the imaging quality. By adding pole pieces of pure iron, we can improve the uniformity of the magnetic field and enlarge the uniformity range. In many papers, as in $[3,4,5,6]$, the design of the magnets was discussed, mostly through optimizing the shape of the pole piece to obtain a more uniform magnetic field. However, in practical engineering, the optimal shapes in theory are complex and difficult to process and produce. To increase the feasibility of the design, we add a shimming ring directly outside the pole piece to improve the uniformity of the magnetic field. GA (genetic algorithms) is used to solve the complex and nonlinear problem in the calculation of the magnetic fields.

We take a small H-type permanent magnet as a model and use the ANSYS software for an electromagnetic field analysis. In addition, the size of the shimming ring outside the pole piece is optimized by GA.

\section{Model and Methods}

Model of Permanent Magnet. The construction of the magnet for MRI consists of magnets, pole pieces, shimming rings and the yoke. As shown in Fig. 1, a small H-type permanent magnet for animal MRI is considered here. For details, see Table 1. 


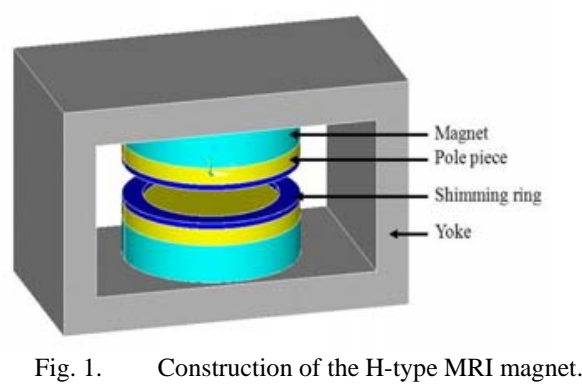

Table 1.
\begin{tabular}{|c|c|c|c|}
\hline Component & Function, Material and Attributes of Permanent Magnet Components \\
\hline Magnet & $\begin{array}{c}\text { Provide the magnetic energy to } \\
\text { generate the static magnetic field }\end{array}$ & $\begin{array}{c}\text { Nd-Fe-B } \\
(\mathrm{N} 40)\end{array}$ & $\begin{array}{c}\mathrm{Br}=1.28 \mathrm{~T} \\
\mathrm{Hcb}=939.0142(\mathrm{kA} / \mathrm{m}) \\
(\mathrm{BH}) \mathrm{max}=300\left(\mathrm{Kj} / \mathrm{m}^{3}\right)\end{array}$ \\
\hline Yoke & $\begin{array}{c}\text { Support the magnet frame to } \\
\text { compose the magnetic circuits }\end{array}$ & A3 Steel & $\mu_{\mathrm{r}}=10000$ \\
\hline $\begin{array}{c}\text { Pole piece\& } \\
\text { Shimming } \\
\text { ring }\end{array}$ & $\begin{array}{c}\text { Smooth the surface of magnets to } \\
\text { get the uniform magnetic field }\end{array}$ & $\begin{array}{c}\text { Pure Iron } \\
\text { DT4 }\end{array}$ & $\mu_{\mathrm{r}}=5730$ \\
\hline
\end{tabular}

Because, in practice, the magnet is composed of many blocks of magnetic material and there are little gaps between the magnetic materials, this leads to the non-uniformity of the magnetic field. The pole piece is made of pure iron on the surface of the magnet and can remarkably smooth the magnetization characteristics of the Nd-Fe-B blocks [7]. However, the surface of the pole piece is flat and the magnetic flux in the air gap is uneven as is the drum; this effect, called the end effect, is shown in Fig.2(a). The most important and effective method is to add a flange outside the pole piece. The flux in the air gap is no longer outside the drum, accordingly, the central magnetic field becomes uniform [8], see Fig.2(b). In practice, to increase the feasibility of processing and producing this system, we add a shimming ring to adjust the field uniformity in the middle of the imaging area, as shown in Fig.2(c). Manufacturers usually determine the size of the shimming ring based on experience, which is a lack of theoretical basis and flexibility. In this paper, we optimize the size of the shimming ring (inner radius $\mathrm{R}_{\mathrm{in}}$ and height $\mathrm{H}$ ) by a GA to provide a reference for the actual production.

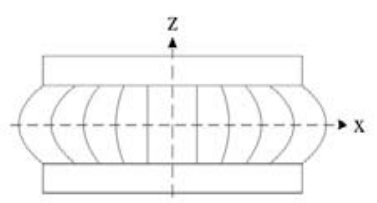

(a)

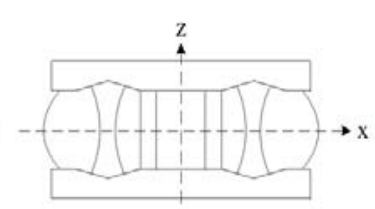

(b)

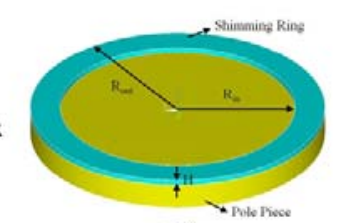

(c)

Fig. 2. (a) Magnetic flux distribution due to the end effect; (b) Magnetic flux distribution with the optimization of the pole pieces; (c) The pole piece model with a shimming ring.

Genetic Algorithm. The GA, developed by John Holland [9] in Michigan University (USA) in the 1960s, is a stochastic optimization method. GA is employed especially to solve complex and nonlinear problems that cannot be readily handled by the traditional search methods [10]. We adopt the optimal preservation strategy in this paper and the flow chart is presented in Fig. 3.

Based on the model of H-type small magnet, we use the GA to adjust the variables of the optimization, namely the inner radius $R_{\text {in }}$ and the height $\mathrm{H}$ of the shimming ring. According to the requirements of the imaging area, we hope to obtain a uniform magnetic field.

(1)Coding and decoding GA cannot directly deal with the parameters of the problem space. They must be transformed into chromosomes or individuals composed by the genes according to the genetic space. In this paper, there are only two variables of optimization, namely, the inner radius $\mathrm{R}_{\text {in }}$ and the height $\mathrm{H}$ of the shimming ring, and they will be encoded by a binary string of 8 bits.

If we transform the variables $X_{m}\left(\mathrm{R}_{\text {in }}\right.$ and $\left.\mathrm{H}\right)$ into an 8-bit binary string $B_{m}\left(B_{1} B_{2} \ldots B_{8}\right)$, the transformation formula from the problem space to the genetic space is established as:

$$
X_{m}=X_{m}^{\min }+\left(X_{m}^{\max }-X_{m}^{\min }\right) /\left(2^{8}-1\right) \sum_{k=1}^{8} 2^{k-1}\left(B_{m}\right)_{k} .
$$

(2)Selection The selection operator is that good individuals are elected and the poor are eliminated based on the fitness of the individual. In this paper, we use the fitness proportional model[11], which is most popularly used in GA. 
The principle of the fitness proportional model is that the selected probability of each individual is proportional to the fitness. Assuming the size of the population is $N$, the individual's fitness is $f_{i}$, therefore the selected probability of individual $P_{s i}$ is:

$$
P_{s i}=f_{\mathrm{i}} / \sum_{j=1}^{N} f_{i} .
$$

The probability $P_{s i}$ indicates the proportion of the individual fitness in the group, and means that the fitter the individual, the higher the probability of being selected. This provides an expression of the natural principle of 'Survival of the fittest'.

Because our optimization goal is to get the minimum uniformity value of the DSV, with the individual being selected based on the maximum fitness, the fitness function is set as:

Fitness $=1 /(1+$ Per $)$,

where Per is the field uniformity in $60 \mathrm{~mm}$ diameter of spherical volume (DSV), defined as

$\operatorname{Per}=\left(B_{\max }-B_{\min }\right) / B_{\text {avg }}$.

$B_{\min }, B_{\max }$ and $B_{a v g}$ are the maximum, the minimum and the average magnetic field intensities of the DSV, respectively. At the same time, we add a constraint that the average magnetic field intensity of the DSV should meet $B_{\text {avg }} \geqslant 0.5 \mathrm{~T}$.

(3)Crossover The crossover operator produces two offsprings by recombining the information from the two parents, which can speed up the process to reach better solutions. It should be in accordance with a certain probability $P_{c}$, range of $0.6-1$. Notable crossover techniques include the single point, the two point, the uniform types [12] and the single point method is adopted in this paper.

(4)Mutation Because the mutation operation is to alter some gene values in an individual at random, meaning that the allele of each gene is a candidate for mutation, its applicability is determined by the mutation probability $P_{m}$. The mutation maintains the diversity of the population to avoid being trapped in a local optimum [13]. The value of the mutation probability $P_{m}$ is usually very small, in the range of 0.001 to 0.1 . For binary coding, the mutation is to negate the value of genes, just as " 0 " to " 1 " or " 1 " to "0".

\section{Results}

In this paper, MATLAB and ANSYS software are working together to program for the simulation and optimization. ANSYS is the commercial finite element method (FEM) analytical software. Because it takes quite a long time to perform the 3D magnetic field computation

Table 2.Comparison of the magnetic fields over the DSV before

\begin{tabular}{|c|c|c|}
\hline \multicolumn{2}{|c|}{ and after the shimming ring optimization. } \\
\hline $\begin{array}{c}\text { Without the } \\
\text { shimming rings }\end{array}$ & 0.5427 & 162.37 \\
\hline $\begin{array}{c}\text { With the optimized } \\
\text { shimming rings }\end{array}$ & 0.5170 & 57.07 \\
\hline
\end{tabular}
in the ANSYS, only half of the magnet was modeled because of the symmetry. The GA procedure is realized by the MATLAB, and the inner radius $\mathrm{R}_{\text {in }}$ and height $\mathrm{H}$ of the shimming ring are transmitted to the ANSYS to simulate the model from the MATLAB. Then, the magnetic field results calculated in ANSYS are passed to MATLAB to be optimized by the GA.

In this paper, the population of the GA was 50; meanwhile the crossover probability is 0.8 and the mutation probability is 0.05 . Finally, we get the optimized size of the shimming ring and the magnetic field results after 50 iterations. The shimming ring after optimization is height $4.5 \mathrm{~mm}$ and inner radius $122.8235 \mathrm{~mm}$. In

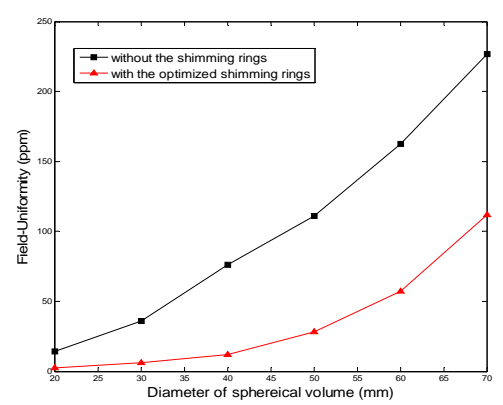

Fig.4. Behavior of the field uniformity variations over the DSV. addition, the comparative results are shown in Table 2.

Although the magnet with the optimized shimming ring produced the magnetic field intensity weaker than without the shimming ring, it is higher than the $0.5 \mathrm{~T}$ that can meet the requirements of 
the design. Moreover, the optimized shimming rings can significantly improve the magnetic field uniformity, which is better by $65 \%$ in the $60 \mathrm{~mm}$ DSV than without the shimming rings. The magnet with the optimized shimming rings maintains a better behavior of field uniformity in the DSV from $20 \mathrm{~mm}$ to $70 \mathrm{~mm}$ than without the shimming rings, see Fig. 4.

\section{Conclusion}

In this paper, the optimal design method using a genetic algorithm was proposed for the design of a small $\mathrm{H}$-type permanent magnet for MRI. The results of the simulation validate the design, and also show that the optimized shimming rings can significantly improve the magnetic field uniformity even though the magnetic field intensity is a little weaker, but within the system requirements. The proposed method based on a genetic algorithm can be widely used to optimize the magnet to generate the homogeneous magnetic field. This can be easily produced in practice.

\section{Acknowledgements}

This work was supported in part by the 973 National Basic Research \& Development Program of China (2010CB732502).

\section{References}

[1] A. Podol'Skii, Design procedure for permanent magnet assemblies with uniform magnetic fields for MRI devices, IEEE Trans Magn, vol.36(02), (2000)484-490.

[2] Dong-Hun Kim, Byung-Sung Kim, Joon-Ho Lee, Wan-Soo Nah, and Il-Han Park, 3-D optimal shape design of ferromagnetic pole in MRI magnet of open permanent-magnet type, IEEE Trans Appl Supercond, vol.12(01), (2002)1467-2002.

[3] L. Sanchez, F. Liu, E. Weber, S. Crozier, Passive shim design and a shimming approach for biplanar permanent magnetic resonance imaging magnets, IEEE Trans Magn, vol.44(03), (2008)394-402.

[4] Tony Tadic, Gino Fallone, Design and optimization of a novel bored biplanar permanent-magnet assembly for hybrid magnetic resonance imaging systems, IEEE Trans Magn, vol. 46(12), (2010)4052-4058.

[5] Tony Tadic, Gino Fallone, Three dimensional non- axisymmetric pole piece shape optimization for biplanar permanent magnet MRI systems, IEEE Trans Magn, vol.47(01), (2011)231-239.

[6] Jae Seop Ryu, Yingying Yao, Chang Seop Koh, Sonam Yun, and Dong Soo Kim, Optimal shape design of 3D nonlinear electromagnetic devices using parameterized design sensitivity analysis, IEEE Trans Magn, vol. 41(05), (2005)1792-1795.

[7] Xiaohua Jiang, Guanghui Shen, Yongchuan Lai, and Jinfeng Tian, Development of an open $0.3 \mathrm{~T}$ NdFeB MRI magnet, IEEE Trans Appl Supercond, vol. 14(02),(2004)1621-1623.

[8] Pingchou Xia. Permanent magnet mechanism. Beijing: Beijing industrial university press. 2000, pp.91-92.

[9] J. H. Holland, Adaptation in Natural and Artificial Systems, Ann Arbor, Michigan: University of Michigan Press, 1975.

[10] Bingzhong Wang. Computational electromagnetics. Beijing: Science Press. 2002, pp.333-334.

[11] De Jong Ka, An analysis of the behavior of a class of genetic adaptive systems, Michigan: University of Michigan, 1975.

[12] W. M. Spears, A. Deiongk, An analysis of multipoint crossover. The Proceeding 1990 Workshop of the Foundations of Genetic Algorithms, (1990)301-315.

[13] William Hoa, George T.S. Hob, Ping Jib, Henry C.W. Lau, A hybrid genetic algorithm for the multi-depot vehicle routing problem, Engineering Applications of Artificial Intelligence, vol. 21(04), (2008)548-557. 